\title{
Treatment
}

\section{Skin to skin contact with their mothers reduced pain reactions in healthy newborn infants during a heel lance}

Gray L, Watt L, Blass EM. Skin-to-skin contact is analgesic in healthy newborns. Pediatrics 2000 Jan;105:e14.

QUESTION: Does mother to infant full body contact reduce pain in newborn infants during a heel lance?

\section{Design}

Randomised (unclear allocation concealment), blinded (assessors of heart rate and crying), controlled trial.

\section{Setting}

A medical centre in Boston, Massachusetts, USA.

\section{Patients}

30 healthy, full term, newborn infants (33-55 hours old, $63 \%$ girls, mean birth weight $3.3 \mathrm{~kg}$ ). None of the infants had evidence of congenital abnormalities, medical complications, or drug exposure, and none required oxygen administration or ventilatory support. This was the initial heel lance for all of the infants. Follow up was complete.

\section{Intervention}

15 infants were allocated to skin to skin contact and 15 were allocated to no contact. 15 minutes before the heel lance, all infants had safety electrodes placed on their thoracic region for heart rate monitoring, and a warming pad was wrapped around the assigned foot. Infants in the no contact group were wrapped in their receiving blankets and placed on their sides in their bassinets. Infants in the contact group wore only a diaper and were positioned, kangaroo style, on their mothers, who were in a comfortable reclined position in their beds, so that skin to skin contact was maintained through the mother's open gown. 2 receiving blankets were placed over the infant's back and mothers were asked to lock their fingers, place their hands over the blankets, and apply a slight pressure on the infant's back. The researchers then left the room for 10-15 minutes so that mother and infant could settle into a relaxed contact position. After a 2 minute baseline period of monitoring, the heel warmer was removed, the heel was swabbed with alcohol, and blood was drawn using a spring loaded lancing device. After blood collection was complete, or after 3 minutes from the start of collection, a bandage was applied to the heel, and the infant entered a 3 minute recovery period.

\section{Main outcome measures}

Behavioural measures of pain were duration of audible crying (assessed from audiotapes) and grimacing (ie, brow bulge, eye squeeze, and nasolabial furrowing assessed from videotapes) during the blood collection and recovery phases. Heart rate was measured every 10 seconds and averaged separately for the baseline, heel stick, and recovery phases.

\section{Main results}

3 infants, for whom data on grimacing could not be ascertained from the videotape, were not included in the analysis. During the blood collection phase, infants who received skin to skin contact cried less (82\% reduction) and grimaced less (65\% reduction) than infants who received no contact. During the 3 minute recovery period, infants who had skin to skin contact cried less (mean $1 v 32 \mathrm{sec}$ ) and grimaced less (mean $2 v 30 \mathrm{sec}$ ) than those who had no contact. Infants in the skin to skin contact group had stable heart rates during the collection and recovery phases (increase of $8-10$ beats $/ \mathrm{min}$ ), whereas infants in the no contact group had a linear increase of 36-38 beats/min to a plateau of 160 beats/min, which was sustained during the first minute of the recovery phase.

\section{Conclusion}

Skin to skin contact with their mother reduced pain reactions in healthy newborn infants during a heel lance.

\section{COMMENTARY}

Until this study by Gray et al, research on skin to skin contact as a non-pharmacological pain intervention for newborns was done on rats and showed that contact induced analgesia. ${ }^{12}$ Although we already know that sucking and sweet taste reduce pain in newborns, ${ }^{3}$ the findings of this study provide an alternative for parents who do not want their infants to suck a pacifier or taste a sweet solution. Taste or sucking are independent of context; contact effectiveness, however, is dependent on 3 factors: firstly, the contact source needs to be a relaxed individual who holds the infant comfortably, yet firmly against her skin; secondly, whereas sucking and taste induced analgesias are of rapid onset, contact induced analgesia is of gradual onset, requiring 10-15 minutes of privacy with the infant in the ventral-ventral position before the heel lance; and thirdly, the mother must be available at the time of the heel lance.

The study participants were 30 healthy, full term infants from 1 institution. The painful procedure was standardised between the 2 groups and the outcome measures included behavioural (cry duration and grimacing), physiological (heart rate), and contextual indicators (eg, behavioural state before the painful procedure). All heel lances were done by the first author of the paper, who was not blinded to whether the infants were in the intervention or control group. To avoid potential bias, it would have been preferable to have had someone less familiar with the study purpose conduct all heel lances.

The results of this study are relevant to paediatric nurses who work with healthy, full term newborns. The study is also relevant to neonatal nurses and neonatal nurse practitioners who work with infants who have many painful procedures, such as repeated heel lances or venipunctures.

The study results emphasise the importance of managing infant pain during painful procedures. For researchers, educators, and clinicians who strive for quality nursing care, skin to skin contact for painful procedures appears to offer promising results for full term infants. Further research with preterm and acutely ill infants is required.

Sharyn Gibbins, RN, MSc Clinical Nurse Specialist/Neonatal Practitioner Neonatal Intensive Care Unit, Women's College Hospital Toronto, Ontario, Canada

1 Blass EM, Watt LB. Suckling- and sucrose-induced analgesia in human newborns. Pain 1999;83:611-23.

2 Blass EM, Shide DJ, Zaw-Mon C, et al. Mother as shield: differential effects of contact and nursing on pain responsivity in infant rats-evidence for nonopioid mediation. Behav Neurosci 1995;109:342-53.

3 Blass EM, Fillion TJ, Weller A, et al. Separation of opioid from nonopioid mediation of affect in neonatal rats: nonopioid mechanisms mediate maternal contact influences. Behav Neurosci 1990;104:625-36. 